\title{
Analysis of the UTexas1 Passive Linear Surface Wave Dataset
}

\author{
Robb Eric S. Moss ${ }^{1}$, Ph.D., P.E., M.ASCE \\ ${ }^{1}$ Dept. Civil and Environmental Engineering, California Polytechnic State University, \\ San Luis Obispo, CA 93407: rmoss@calpoly.edu
}

\begin{abstract}
A linear array consisting of twenty-four $1 \mathrm{~Hz}$ geophones at $10 \mathrm{~m}$ spacing was used to measure the passive surface waves at the UTexas1 site. This paper describes analysis of this dataset using three different methods: ESPAC (extended spatial autocorrelation), f-k (frequency-wavenumber), and ReMi (refraction microtremor). Dispersion curves were developed using each method and the median trends as well as the uncertainty about the medians are compared. The dispersion curves were then individually inverted to estimate shear wave velocity profiles. The inversion results are compared, and a bounded best-estimate shear wave velocity profile is presented. For the site conditions, specific recording equipment, and array geometry the ESPAC method was the most consistent between recordings, and able to resolve the lowest frequency Rayleigh waves. The shear wave velocity of all three methods were in close agreement in the upper $20 \mathrm{~m}$ above a stiff layer, but were increasing disparate as depth increased. All three methods resolved a velocity inversion, a stiff layer, at roughly $20 \mathrm{~m}$ to $55 \mathrm{~m}$ depth overlying softer material. As is expected with surface wave methods, and particularly with passive methods that are measuring ambient noise along single linear array, the uncertainty in the dispersion curves increased with decreasing frequency, and the uncertainty in the shear wave velocity profiles increased with increasing depth.
\end{abstract}

\section{PASSIVE SURFACE WAVE ANALYSIS}

The ambient noise present at a particular site is the source for passive wave measurements. The broader the spectrum and the lower the frequency the better will be the imaging of the subsurface conditions. A typical ambient noise spectrum for this UTexsas1 site (Figure 1) shows broad response up to roughly $15 \mathrm{~Hz}$ with a spike around $27.5 \mathrm{~Hz}$.

The first method used to analyze the passive linear recordings was ESPAC (extended spatial autocorrelation). The concept for SPAC was originally proposed by Aki (1957) who suggested that a low order bessel function can be fit to recordings from a circular array to evaluate the coherency of the ambient waves across the array. 


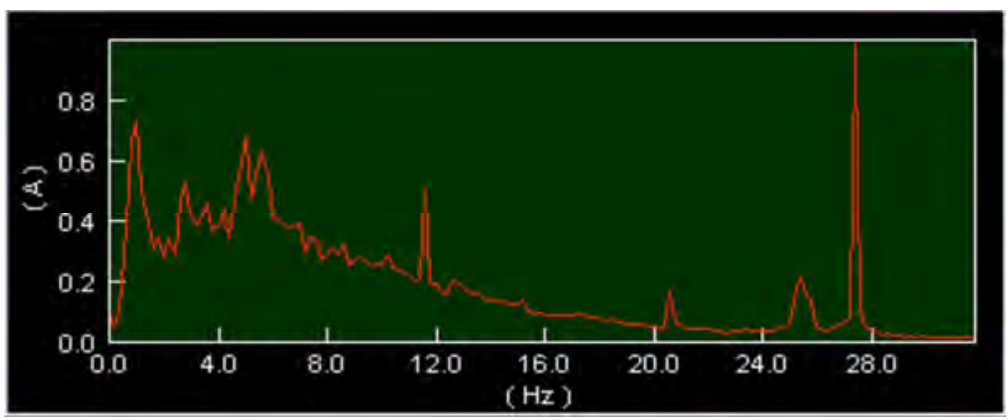

Fig. 1. Typical ambient spectrum for the linear passive array showing the frequency $(\mathrm{Hz})$ versus normalized amplitude (A) shown to the left.

This method was extended to applications where the array is linear and has been coded into the software Surface Plus by Geogiga (http://www.geogiga.com accessed 6/1/13). All 10 recordings from the linear passive array were analyzed using ESPAC, and recordings 1 through 7 were selected for showing the "clearest" dispersion or Rayleigh wave phase velocity versus frequency trends. These were combined into a single recorded dispersion plot with a $30 \mathrm{~Hz}$ cutoff (Figure 2).
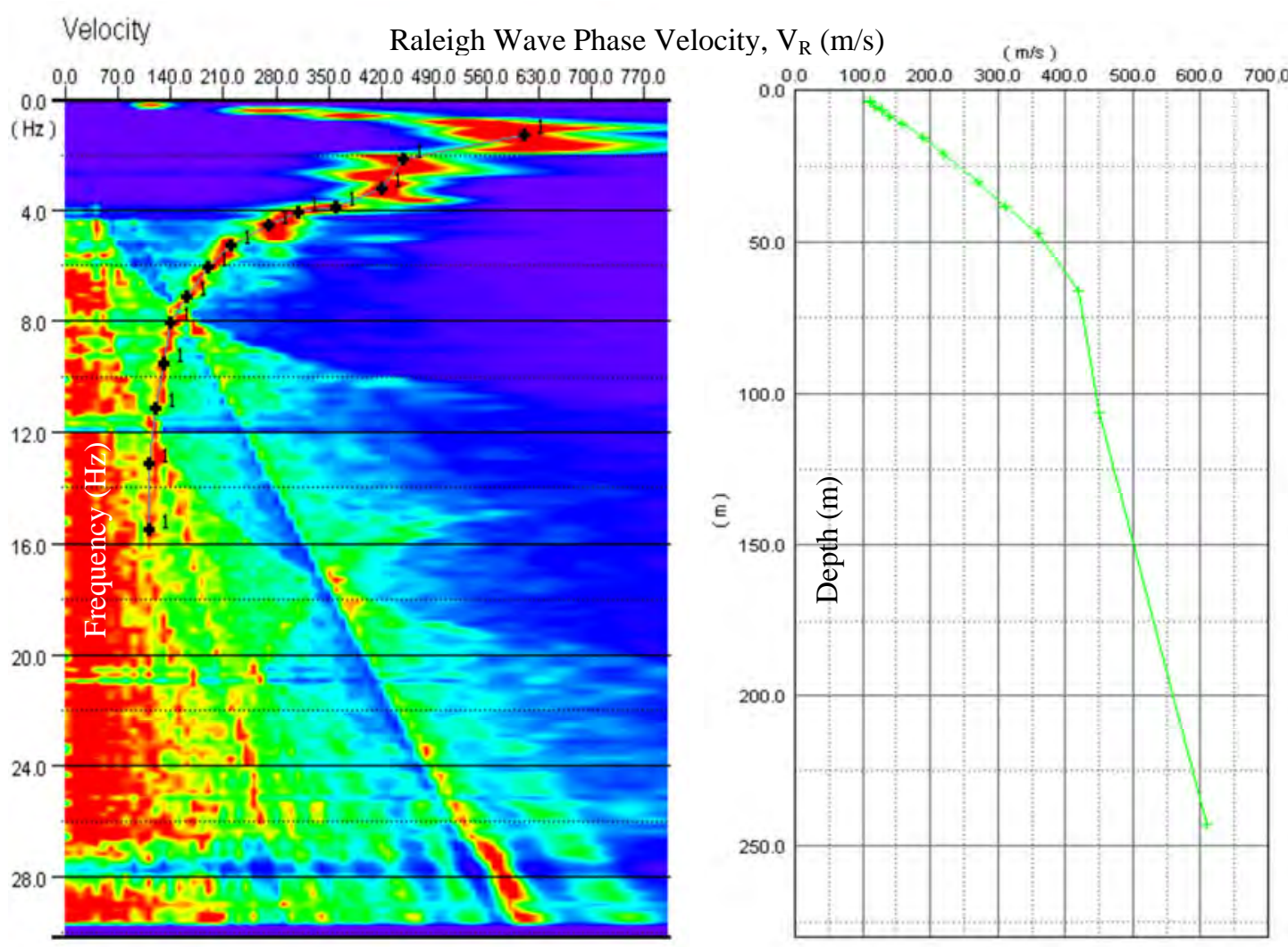

Fig. 2. Left plot shows the ESPAC measured dispersion (Rayleigh wave phase velocity versus frequency) with manually picked dispersion trend. The right depth velocity (HV) plot shows the picked Rayleigh wave phase velocity versus estimated depth.

The color map in Surface Plus was adjusted to give the best appearant resolution, balancing the strong signature in the lower frequencies $(1-3 \mathrm{~Hz})$ with the weaker signature in the higher frequencies $(10-14 \mathrm{~Hz})$. The resulting combined recorded 
dispersion curve guided the manual picking of a median dispersion trend. The depth versus velocity plot is an estimate that assumes depth equals one half the wavelength.

The manually picked dispersion curve can be used as a first-order estimate of the $\mathrm{V}_{\mathrm{S}}$ profile (Figure 2) by assuming a Poisson ratio of 0.5, thereby producing a ratio between Rayleigh wave phase velocity and shear wave velocity of $\mathrm{V}_{\mathrm{R}} / \mathrm{V}_{\mathrm{S}} \sim 0.95$ (Stokoe et al., 2004). The site appears relatively normally dispersive, except for a "kink" in the dispersion curve. This "kink" can be better modeled using an inversion procedure to estimate the shear wave velocity profile.

To estimate the shear wave velocity profile an inversion procedure is typically employed. This iterative approach attempts to find the "best" shear wave velocity profile that agrees with dispersion trend. The soil layers can be modeled both in depth extent and magnitude of velocity. Geogiga uses a genetic algorithm to find the "best fit" with a number of user controlled options to assist in the fitting procedure. The modeled fit does a reasonably good job accommodating the "kink" around $3 \mathrm{~Hz}$ while still fitting the rest of the observed dispersion curve (Figure 3).

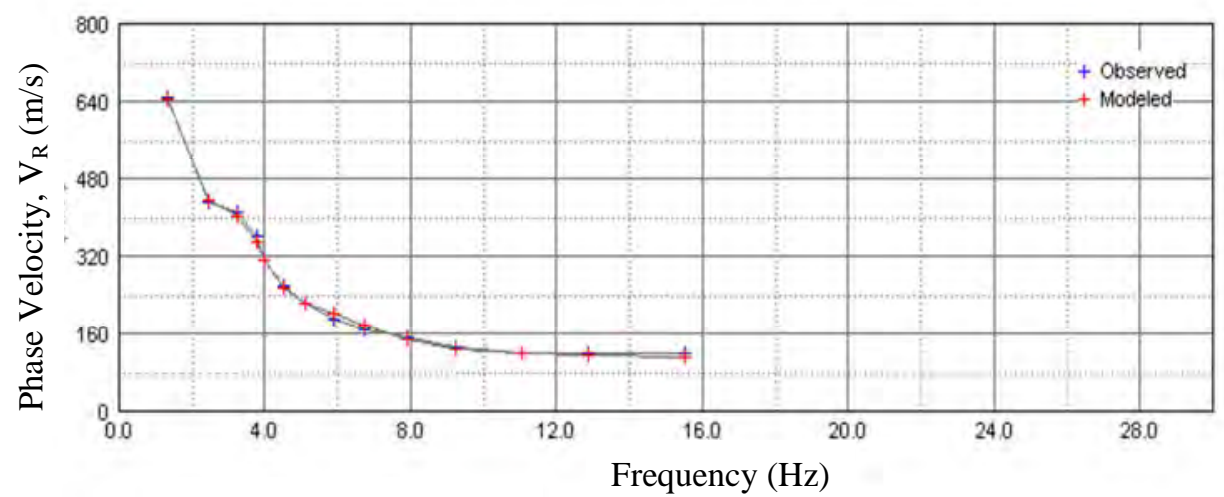

Phase Velocity, $\mathrm{V}_{\mathrm{R}}(\mathrm{m} / \mathrm{s})$

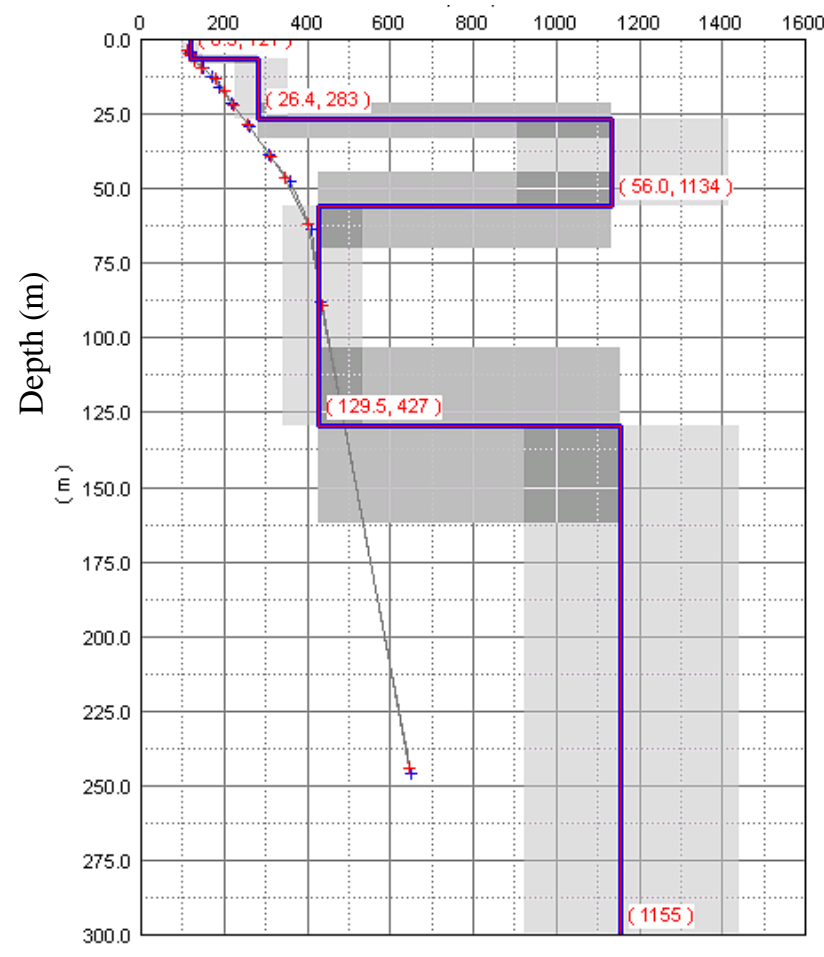

Fig. 3. Inverse modeling of the picked dispersion trend from ESPAC method using Geogiga Surface Plus. Top plot shows frequency versus Rayleigh wave phase velocity, with the observed/picked trend versus the modeled trend. Plot to the left shows the shear wave velocity versus depth profile with shaded uncertainty ranges. 
The velocity at the bottom of the profile is controlled by the low frequency (long wavelength) waves where the dispersion resolution and inversion sensitivity are at odds. The resulting shear wave velocity estimate is shown with a clear velocity inversion. Surface Plus provides estimates of the uncertainty in depth and velocity shown as shaded bands around the median lines.

The f-k (frequency wavenumber) method can also be applied to the linear array, although this method is known to work best at higher energies. The f-k method uses an analysis of the power spectrum to estimate the dispersion curve and was developed and applied by Capon (1969), Asten and Henstridge (1984), and Tokimatsu et al., (1991) among others. All 10 recordings were analyzed and the "cleanest" measured dispersion trends from recordings 3,4 , and 5 were combined into the composite measured dispersion where the color palate was optimized for manually picking the dispersion trend (Figure 4).

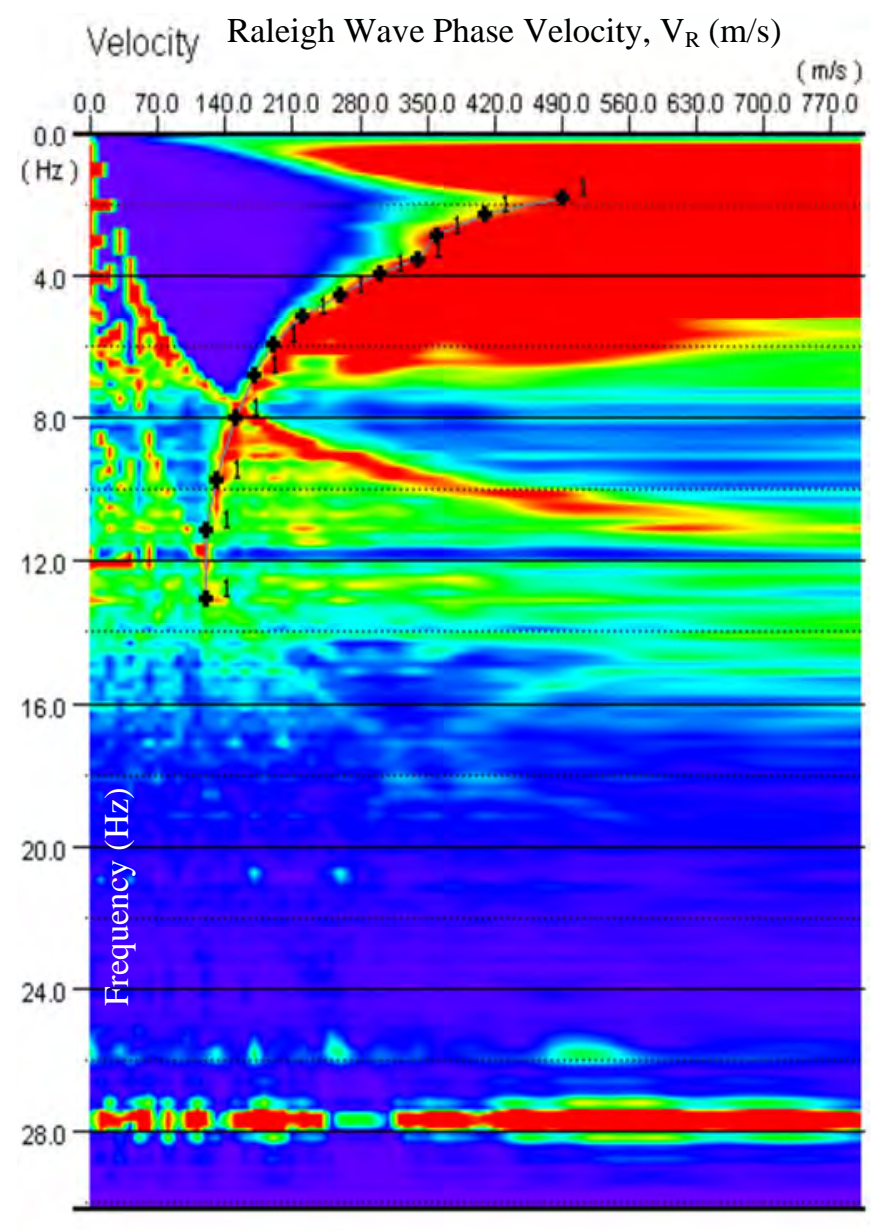

Fig. 4. Measured dispersion based on the f-k method. Manual dispersion curve estimates are shown as black plus signs .

The modeled versus observed dispersion curve is shown in Figure 5 with a similarly reasonable fit as was achieved with the ESPAC analysis. The estimated shear wave velocity profile was inverted using the same genetic algorithm applied to the f-k analysis. For the inversion process in all these methods presented, if the algorithm attempted to fit a layer that was obviously too thin for the given geophone spacing $(10 \mathrm{~m})$ then that layer was lumped with an adjacent layer to provide a more justifiable profile. 

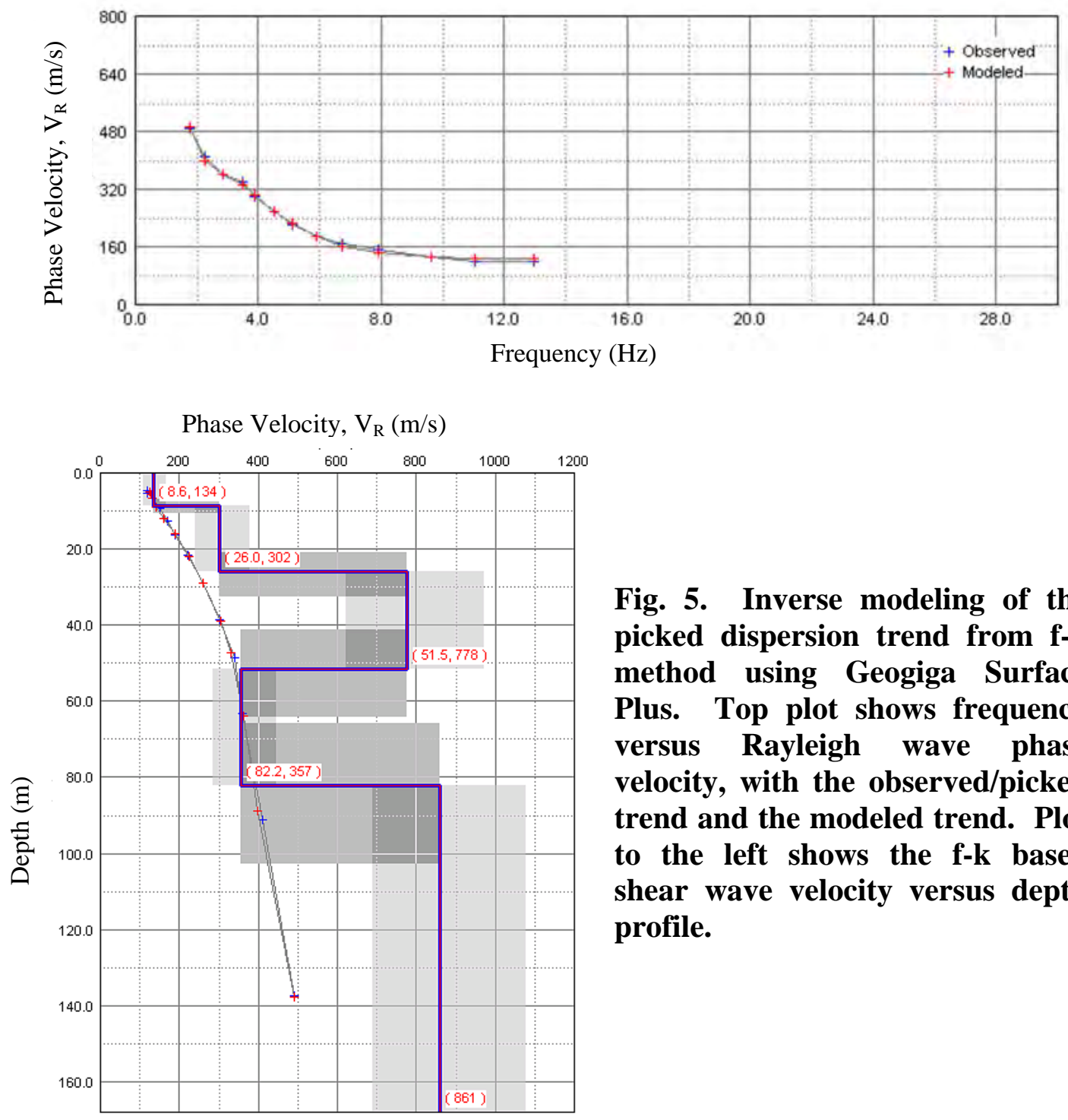

Fig. 5. Inverse modeling of the picked dispersion trend from f-k method using Geogiga Surface Plus. Top plot shows frequency versus Rayleigh wave phase velocity, with the observed/picked trend and the modeled trend. Plot to the left shows the $f-k$ based shear wave velocity versus depth profile.

ReMi (refraction microtremor), the third method used in this study was applied to this linear array with the Optim software ReMiVspec and ReMiDisper (http://www.optimsoftware.com/ accessed 6/1/13). Out of the 10 recordings (here numbered as 0 to 9 ) the records $0,2,3,4$, and 5 were combined into one frequency versus slowness plot for manually picking the observed dispersion trend. Multiple points were picked along the lower bound as recommended by Louie et al. (2001).

The Optim software, similar to Geogiga Surface Plus, uses a genetic algrorithm to invert the shear wave velocity profile, fitting the observed dispersion picks with a theoretical dispersion curve. This provided a reasonable overall fit, and accomodated the "kink" in the dispersion curve adaquetly (Figure 7). Initial parameters were varied but the resulting $\mathrm{V}_{\mathrm{S}}$ profiled was relatively uninfluenced. 


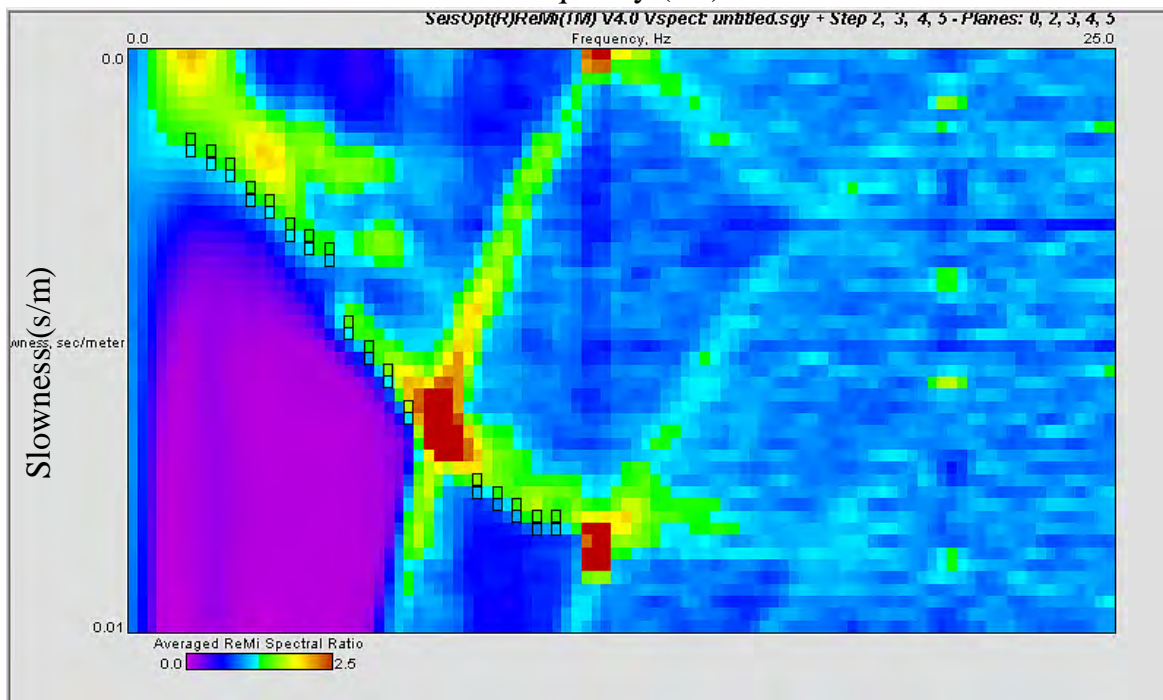

Fig.6.

Frequency

versus

slowness

(1/velocity)

plot using

ReMi

method.

Dispersion

picks shown

as open

squares.

A second manual dispersion pick was performed with a denser set of picks. The more picks resulted in more difficulty fitting a simple model. The automatic inversion program produced 10 to 12 layer models with thin layer that could not realistically be resolved using the $10 \mathrm{~m}$ spacing of the geophones.

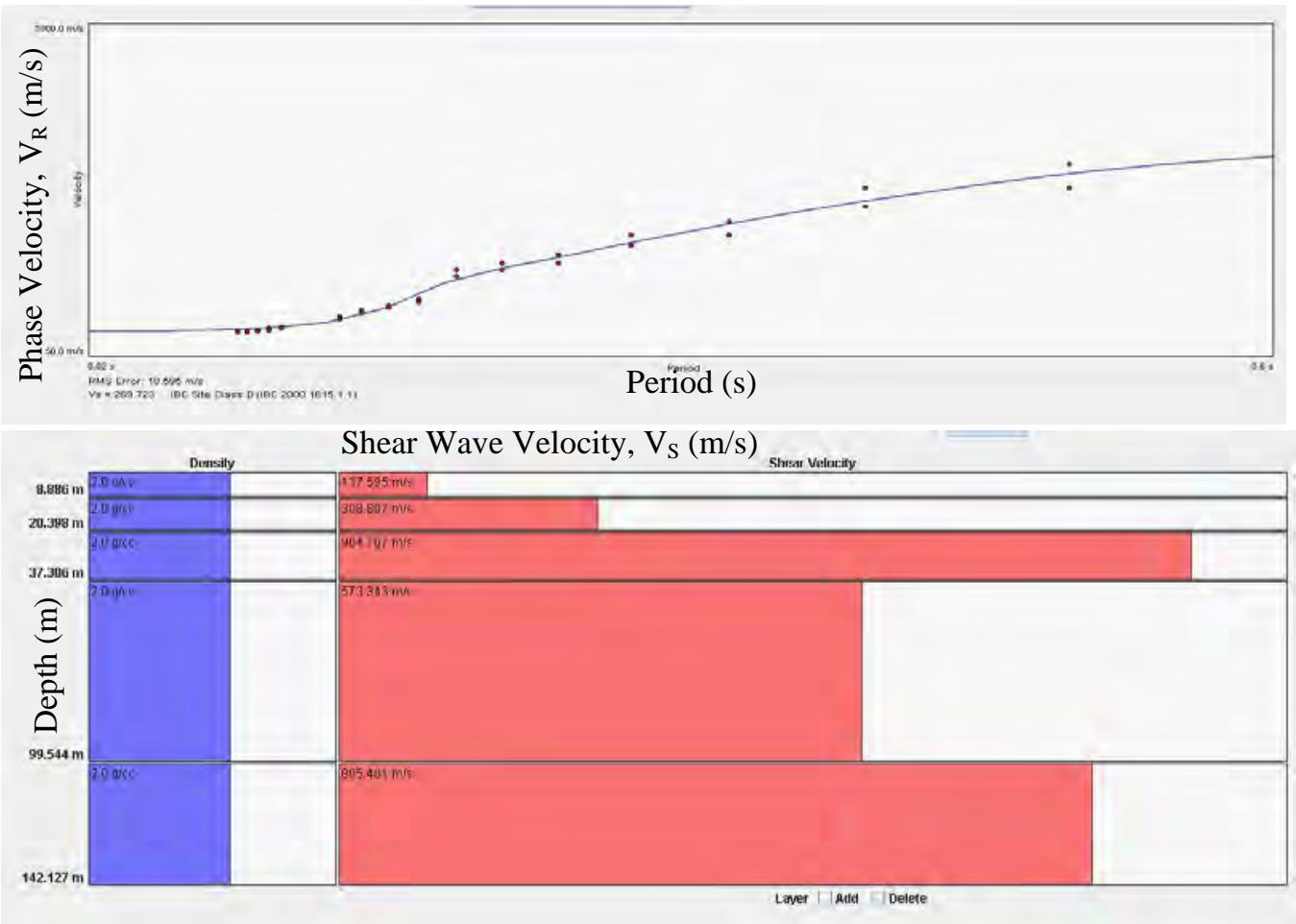

Fig. 7. Inverse modeling of the picked dispersion trend from ReMi method using Optim. Top plot is frequency versus Rayleigh wave phase velocity, with the observed/picked trend and the modeled trend. Bottom plot is the ReMi based shear wave velocity versus depth profile. 


\section{COMPARISON OF RESULTS}

All three methods, ESPAC, f-k, and ReMi, provided consistent dispersion trends as can be seen in Figure 8; although there is some discrepancy as to what frequency range the "kink" occurs at in the dispersion curve. The lowest frequency that can be resolved is very much a function of the analysis method, with ESPAC sampling the lowest and therefore imaging the deepest. ESPAC also provided the broadest resolution from high to low frequencies (shallow to deep layers) and is thought to represent the median trend reasonably well. The three methods resolved the stiff layer at different frequencies with ReMi imaging the "kink" around $5 \mathrm{~Hz}$, ESPAC around $3 \mathrm{~Hz}$, and $\mathrm{f}-\mathrm{k}$ in between.

Fig. 8.

Comparison of dispersion picks for the three passive analysis methods used in this analysis shown in the figure to the right.

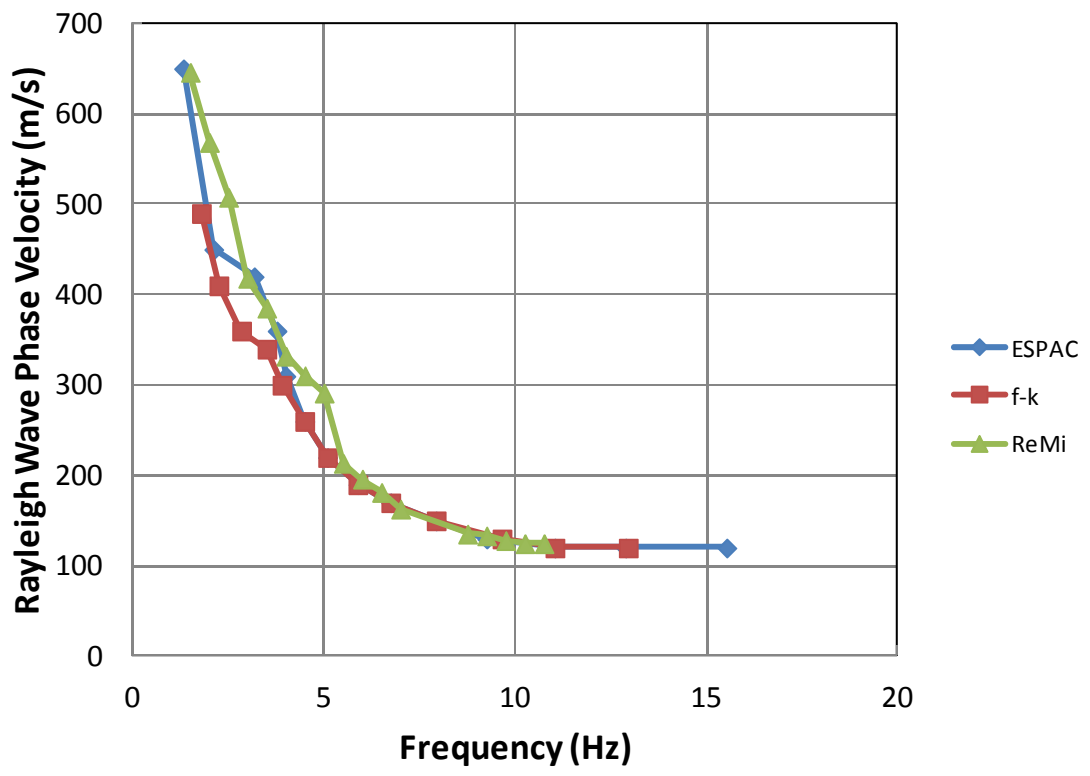

A comparison of the inverted shear wave velocity profiles is shown in Figure 9. As can be seen there is increasing disagreement with depth of the velocity for each layer and the depths of each layer interface. In general all three methods resolved a stiff layer between 20 to 55 meters depth, and a softer layer below the stiff layer. There is good agreement in the near surface, above the stiff layer (0 to $20 \mathrm{~m}$ ) as would be expected of surface wave methods. There is poor agreement in the velocity of the stiff layer, the soft layer directly below, and the depths of these layer boundaries.

Table 1 shows the values of $\mathrm{V}_{\mathrm{S} 30}$ for this site per the method used. There is not an appreciable difference, as the stiff layer starts around the $30 \mathrm{~m}$ mark, and the methods show good agreement above 20 to $25 \mathrm{~m}$. From a site response perspective the differences in $\mathrm{V}_{\mathrm{S} 30}$ are nominal. However with the velocity inversion, this site is likely to have strong response at modes other than the first mode, which would not be reflected in the $\mathrm{V}_{\mathrm{S} 30}$. 
Fig. 9. Comparison of estimated shear wave velocity profiles shown in the figure to the right.

Table 1. $\begin{gathered}\text { V } \\
\text { S30 values } \\
\text { from different } \\
\text { methods }\end{gathered}$
\begin{tabular}{|l|c|}
\hline Method & $\begin{array}{c}\mathbf{V}_{\text {S30 }} \\
(\mathbf{m} / \mathbf{s})\end{array}$ \\
\hline ESPAC & 240 \\
\hline f-k & 236 \\
\hline ReMi & 268 \\
\hline
\end{tabular}

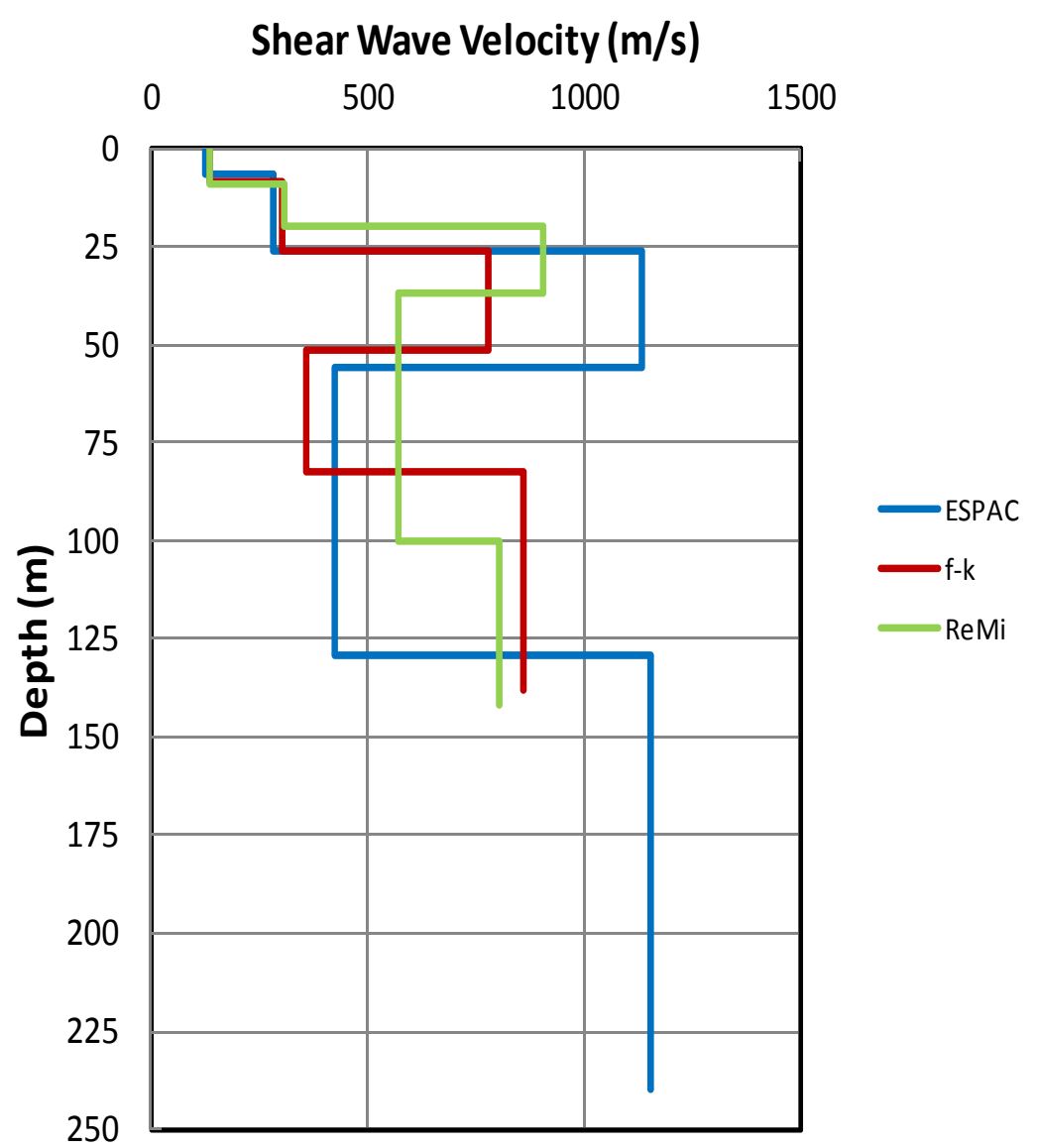

\section{PASSIVE SURFACE WAVE TESTING UNCERTAINTY}

The uncertainty in the dispersion curves vary as a function of the frequency. The range of Rayleigh wave phase velocity values at the lowest frequencies (hence the deeper layers) is on the order of $100 \mathrm{~m} / \mathrm{s}$, irrespective of the analysis method used. The range of values at the highest frequencies (hence the shallowest layers) is on the order of $10 \mathrm{~m} / \mathrm{s}$, irrespective of the analysis method. A representative dispersion plot, Figure 10, based on median ESPAC results shows this range with frequency. The range is based on multiple dispersion trend picks or analysis iterations for each method, and the sampling resolution with frequency of surface wave testing. The longer the wavelength, the lower the frequency, the poorer the resolution as measured using surface waves. The range here represents a true range of realized dispersion values for a single analysis method.

The range of median values in the shear wave velocity profile is shown in Figure 11. Here the median values from the three methods bound a best estimate of the velocity with depth. This range ignores the upper and lower bound uncertianty for each method and only represents the range of medians between methods. The upper $20 \mathrm{~m}$ are well characterized, with uncertainty blossoming at and below the stiff layer. 


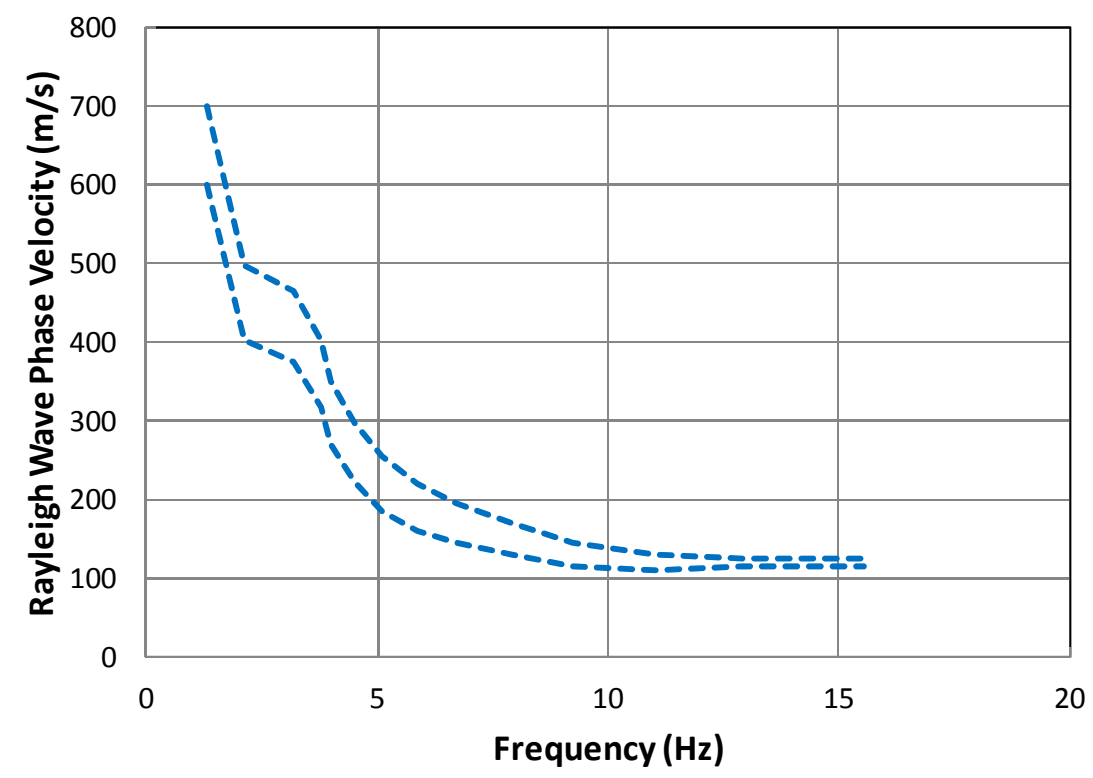

Fig. 10.

Uncertainty range of dispersion curves showing a decrease in uncertainty as frequency increases shown in figure to the left.

Fig. 11. Best-estimate shear wave velocity profile ranges, both in velocity and depth shown in figure to the right.

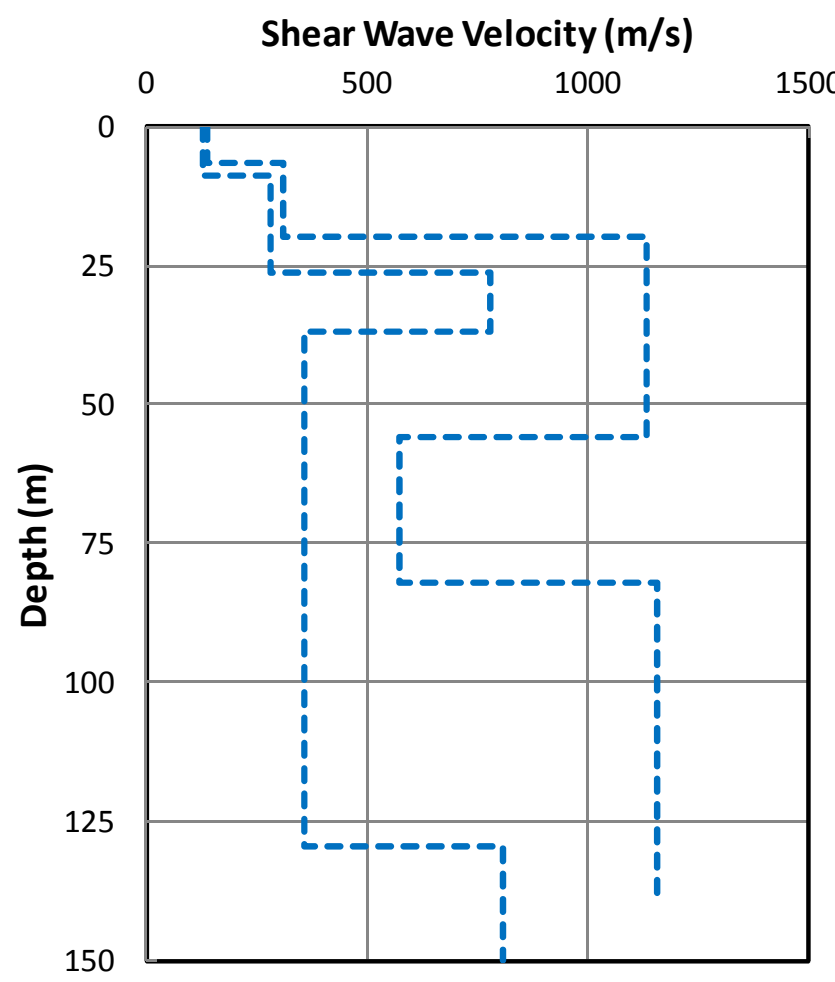

The shear wave velocity estimates had a range of $10 \mathrm{~m} / \mathrm{s}$ near the ground surface and a range of $300 \mathrm{~m} / \mathrm{s}$ for the stiff layer at $20 \mathrm{~m}$ to $55 \mathrm{~m}$ depth. Layer depth resolution had a range of less than $5 \mathrm{~m}$ near the ground surface and upwards of $30 \mathrm{~m}$ at depth.

\section{SUMMARY AND RECOMMENDATIONS}

Three commonly employed methods were used to analyze the passive linear array. ESPAC resolved the broadest range of frequencies, particularly at lower frequencies, which are sampling deeper in the profile. f-k provided dispersion trends similar to the ESPAC method but with less consistency (i.e., fewer number of recordings that were "clean") and was limited in the low frequencies. ReMi results were similar to f-k but covered a broader frequency range. 
All methods used a genetic algorithm to invert a shear wave velocity profile to the observed dispersion curve picks. The uncertainty in this process increases with decreasing frequency and hence increasing depth. It is suggested that future inversion algorithms use an approach that constrains the fit with a top-down procedure, fitting shallow first then using that information to proceed deeper to help constrain the increasing uncertainty. It would also be useful if a minimum layer thickness criteria were applied in automated inversion algorithms to eliminate unrealisticly thin layers in the solution.

For these particular site conditions, array geometry and equipment, and passive noise, ESPAC provided the broadest resolution of Rayleigh wave phase velocity dispersion thereby sampling the deepest. These results are similar to other studies where SPAC/ESPAC provide the most consistent results from recording to recording and can resolve the broadest frequency spectrum from ambient noise (e.g., Richwalsky et al., 2007; Hunter et al., 2012). For future sites where the conditions are similarly unknown it is recommended that ESPAC be used to analyze the passive data. As in all cases of passive surface wave testing the more methods that are used, the more confidence there is in the estimated dispersion curve and inverted shear wave velocity profile, so it is recommended that SPAC/ESPAC be used regularly in conjunction with other methods.

\section{REFERENCES}

Aki, K. (1957). "Space and time spectra of stationary stochastic waves, with special reference to microtremors” BRRI, v. 35, p. 415-456.

Asten, M.W. and Henstridge, J.D., (1984). "Array estimators and the use of microseisms for reconnaissance of sedimentary basins.” Geophys., v.49, p.18281837.

Capon, J., (1969). "High-resolution frequency-wavenumber spectrum analysis." Proc., IEEE, v. 57, p.1408-1418.

Hunter, J.A., and Crow, H.L. (eds.) et al. (2012). Shear Wave Velocity Measurement Guidelines for Canadian Seismic Site Characterization. Geologic Survey of Canada, Open File 7078.

Louie, J.N. et al., (2001) "Faster, better: shear wave velocity to 100 meter depth from refraction microtremor arrays.” BSSA, 91, 347-364.

Richwalski, S. M., et al., (2007). "Rayleigh wave dispersion curves from seismological and engineering geotechnical methods.” J. of Geophysics and Engineering, Vol. 4, Num. 4.

Stokoe, K.H., Joh, S.H., and Woods, R.D., (2004). "Some Contributions on In Situ Geophysical Measurements to Solving Geotechnical Engineering Problems.” Proc. ISC- 2 Geotech. \& Geophys. Site Characterization, Sept 19- 22, Porto, Portugal, v. 1, p. 97-132.

Tokimatsu, K., Shinzawa, K., and Kuwayama, S. (1992). "Use of Short Period Microtremors for Vs Profiling.” J. Geotechnical Engineering,118(10), 1544-1558. 